\title{
O problema probe particionado split bem-coberto é polinomial
}

\author{
S. R. Alves ${ }^{1}$, F. Couto ${ }^{2}$, L. Faria ${ }^{3}$, S. Klein ${ }^{4}$, U. dos S. Souza ${ }^{5}$ \\ ${ }^{1}$ FAETEC/RJ - Rio de Janeiro, ${ }^{2}$ UFRRJ - Nova Iguaçu, ${ }^{3}$ UERJ - Rio de Janeiro \\ ${ }^{4}$ UFRJ - Rio de Janeiro, ${ }^{5}$ UFF - Niterói \\ Brazil \\ sancrey@gmail.com, nandavdc@gmail.com, luerbiodcos.ufrj.br, \\ sula@cos.ufrj.br, uevertonssouza@gmail.com
}

\begin{abstract}
A graph $G=(V, E)$ is well-covered if each maximal independent set of $G$ is maximum. A graph $G=(V, E)$ is split if there is a partition $V=(S, K)$, where $S$ is an independent set and $K$ is a clique. A well-covered split graph is at a same time well-covered and split. Given a class $\mathcal{C}$ of graphs, a graph $G=(V, E)$ is $\mathcal{C}$-probe if there is a partition for $V=(N, P)$ into probes $P$ and non-probes $N$, where $N$ is an independent set and new edges may be added between non-probes such that the resulting graph is in the graph class $\mathcal{C}$. We say that $(N, P)$ is a $\mathcal{C}$ probe partition for $G$. The $\mathcal{C}$-PARTITIONED PROBE problem consists of an input graph $G$ and a probe partition $V=(N, P)$ and the question: Is $(N, P)$ a $\mathcal{C}$-probe partition? In this paper we consider $\mathcal{C}$ the class of well-covered split graphs, and we prove that $\mathcal{C}$-PARTITIONED PROBE problem belong to the polynomial problem class $P$.
\end{abstract}

Resumo. Um grafo $G=(V, E)$ é bem-coberto se cada conjunto independente maximal de $G$ é máximo. Um grafo $G=(V, E)$ é split se existe uma partição $V=(S, K)$, onde $S$ é um conjunto independente e $K$ é uma clique. Um grafo split bem-coberto é, ao mesmo tempo, split e bem-coberto. Dada uma classe $\mathcal{C}$ de grafos, um grafo $G=(V, E)$ é $\mathcal{C}$-probe se existe uma partição para $V=(N, P)$ em probes $P$ e não-probes $N$, onde $N$ é um conjunto independente e novas arestas podem ser adicionadas entre não-probes de maneira que o grafo resultante permaneça na classe de grafos $\mathcal{C}$. Dizemos que $(N, P)$ é uma $\mathcal{C}$ probe partição para $G$. O problema $\mathcal{C}$-PROBE PARTICIONADO consiste de um grafo de entrada $G$ e uma partição probe $V=(N, P)$ e a questão: $(N, P)$ é uma $\mathcal{C}$-partição probe? Neste artigo, consideramos $\mathcal{C}$ como a classe dos grafos split bem-cobertos, e provamos que o problema $\mathcal{C}$-PROBE PARTICIONADO pertence à classe de problemas polinomiais $P$.

Dado um grafo $G=(V, E)$, um vértice $u$ é adjacente a $v$ se $u v \in E(G)$. Dado $S \subset V$, o conjunto $N_{S}(u)=\{\forall v \in V(G): u v \in E\}$ é chamado de vizinhança aberta de $u$ em $S$. Denotamos $N(v)$ a vizinhança aberta de $v$ em $G$. $H$ é um subgrafo de $G$ se $V(H) \subseteq V(G)$ e $E(H) \subseteq E(G)$. Uma clique $K \subset V$ é tal que para cada par de vértice $u, v \in K, u v \in E$. Um conjunto independente $S \subset G$ é tal que para cada par de vértice $u, v \in S, u v \notin E$. Um conjunto $S \subset V$ é um conjunto independente maximal de $G$, se não há conjunto independente de $G$ contendo propriamente $S$. Um grafo é split quando seu conjunto de vértices pode ser particionado em uma clique e um conjunto independente. Um grafo $G$ é dito bem-coberto se cada conjunto independente 
maximal é máximo. Embora o o problema de reconhecimento BEM COBERTO seja coNPcompleto [Chvátal and Slater 1993] para grafos em geral, provamos que o problema de reconhecimento SPLIT BEM-COBERTO [Alves et al. 2016] é um problema polinomial.

Teorema 1 [Alves et al. 2016] Um grafo G é um grafo split bem-coberto se, e somente se, admite uma partição split bem coberta $V=(S, K)$ onde $S$ é um conjunto independente e $K$ uma clique tal que, ou $\forall x \in K,\left|N_{S}(x)\right|=0$, ou $\forall x \in K,\left|N_{S}(x)\right|=1$.

Os vértices $u \in S$ adjacentes a vértices $v \in K$ são ditos testemunhas de $v$. Assim, o Teorema 1 garante, em outras palavras, que um grafo é split bem-coberto se, e somente se admite uma partição de $V$ em um conjunto independente $S$ e uma clique $K$, tal que, ou os vértices de $K$ não têm testemunhas, ou cada vértice de $K$ tem exatamente uma testemunha. Golumbic, Kaplan e Shamir [Golumbic et al. 1995] provaram que PROBE PARTICIONADO SPLIT é polinomial. Nesta seção propomos um algoritmo polinomial para o problema de decisão PROBE PARTICIONADO SPLIT BEM-COBERTO.

PROBE PARTICIONADO SPLIT BEM-COBERTO - (PPSPLIT-WC)

InSTÂNCIA: Grafo $G=(V, E)$ e uma partição probe $V=(N, P)$, onde $N$ é um conjunto independente de $G$.

PERGUNTA: Existe um conjunto $E^{\prime} \subseteq N \times N$ tal que $H=\left(V, E \cup E^{\prime}\right)$ é um grafo split bem-coberto?

Teorema 2 Se a instância $(G=(V, E), N, P)$ é sim para o problema PROBE PARTICIONADO SPLIT, então $G$ é split e uma solução com o grafo split bem-coberto $H$ com partição split bem coberta $(S, K)$ é tal que que a partição split $P=\left(P_{S}, P_{K}\right)=(P \cap S, P \cap K)$ de $P$ é uma das $O\left(n^{2}\right)$ possíveis partições de $P$ do grafo split $H$.

O Algoritmo 1 proposto utiliza o conceito de partição esparso-denso proposto por [Feder et al. 1999], precisamente no fato que existe um número polinomial $O\left(n^{2}\right)$ de partições esparso-densas $(S, K)$ de um grafo split. Mais ainda, uma vez conhecendo uma, as demais podem ser encontradas em tempo polinomial. Nosso algoritmo vai olhar para cada uma dessas partições uma única vez. Assim, dada uma partição split obtida em tempo polinomial [Golumbic 1980] para o grafo de entrada $G$ e, dada $P=\left(P_{S}, P_{K}\right)$ uma partição split esparso-densa [Feder et al. 1999] obtida a partir da partição inicial split para $G$, onde $P_{S}$ é um conjunto independente e $P_{K}$ é uma clique de $G$, observamos que $N$ pode ser particionado em dois conjuntos: $N_{S}=\{u \in N$ : existe $u v \notin E$ tal que $\left.v \in P_{K}\right\}$ e $N_{K}=\left\{u \in N: u v \in E\right.$ para todo $\left.v \in P_{K}\right\}$. Nós consideramos cada um dos possíveis casos: $\left(N_{S}, N_{K}\right)=(\emptyset, \emptyset),\left(N_{S}, N_{K}\right)=(\emptyset, \neq \emptyset),\left(N_{S}, N_{K}\right)=(\neq \emptyset, \emptyset)$, e $\left(N_{S}, N_{K}\right)=(\neq \emptyset, \neq \emptyset)$. A seguir, explicaremos cada um destes casos bem como as respectivas tomadas de decisão. Em nossa notação $K(S), S \subset V$ significa o grafo obtido de $G[S]$ pela adição de todas as arestas entre os vértices de $N \cap S$.

Para a corretude do Algoritmo 1, vamos analisar os seguintes casos:

1. $G$ é split bem-coberto. Caso trivial que contempla os casos: $\left(N_{S}, N_{K}\right)=(\emptyset, \emptyset)$ e $\left(N_{S}, N_{K}\right)=(\neq \emptyset, \emptyset)$ descritos acima. Com partição split bem coberta $\left(N_{S} \cup\right.$ $\left.P_{S}, N_{K} \cup P_{K}\right)=(S, K)$.

2. $N_{S}=\emptyset, N_{K} \neq \emptyset$ e $P_{S}=\emptyset$. Observe que, basta adicionar todas as arestas possíveis entre os vértices de $N_{K}$ que teremos um grafo split bem-coberto com partição split bem coberta $(S, K)=(\emptyset, K)$ onde cada vértice de $K$ tem zero vizinhos em $S$. 


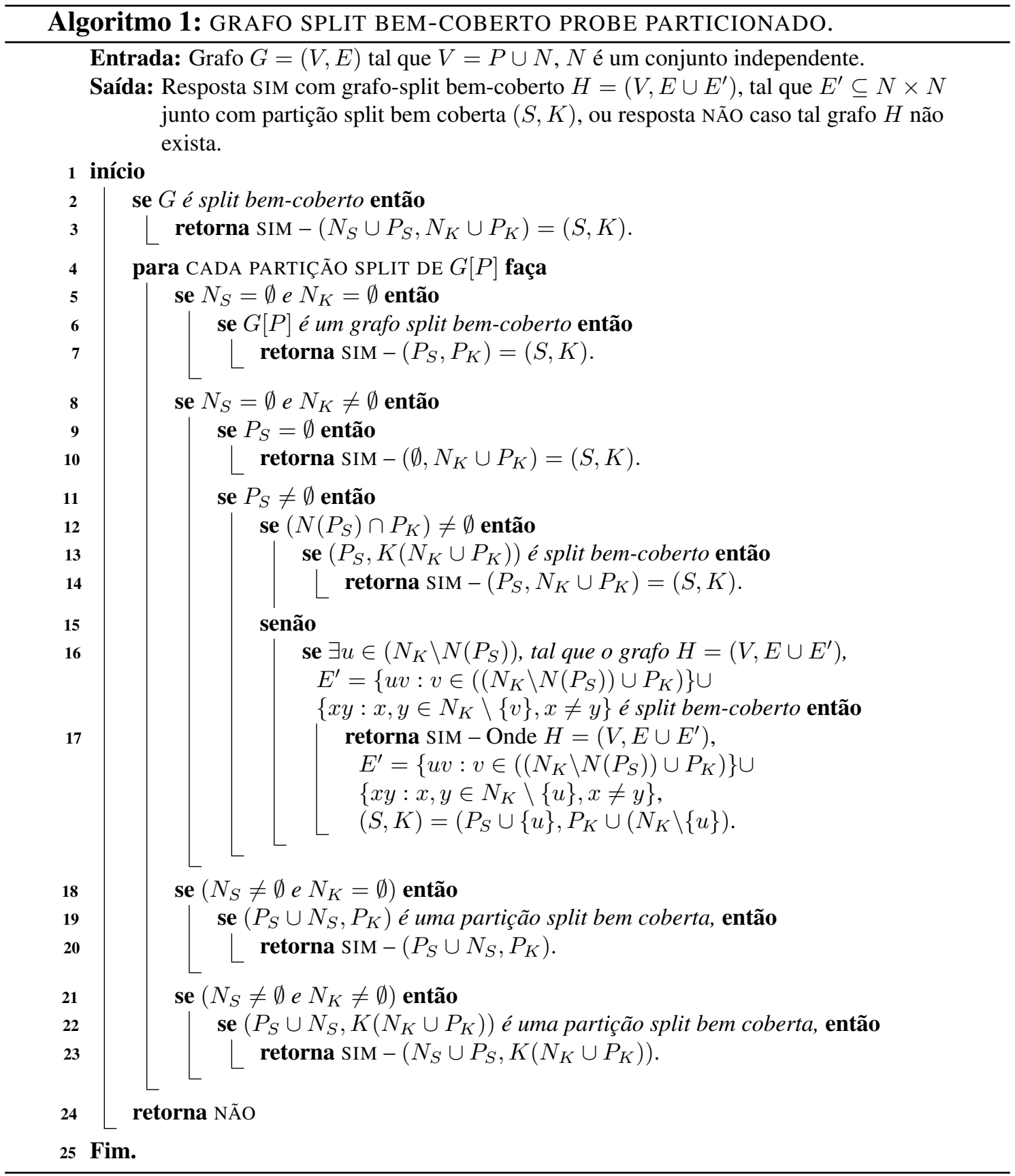

3. $N_{S}=\emptyset, N_{K} \neq \emptyset, P_{S} \neq \emptyset$ e $\left(N\left(P_{S}\right) \cap P_{K}\right) \neq \emptyset$. Uma vez que existe pelo menos um vértice em $P_{S}$ com adjacência em $P_{K}$, pelo Teorema 1 , é necessário que cada um dos vértices de $P_{K}$ tenha exatamente um vizinho em $P_{S}$. Primeiramente, note que as testemunhas dos vértices de $P_{K}$ devem pertencer apenas a $P_{S}$, caso contrário, pela definição de $N_{S}$, um vértice em $P_{K}$ teria mais de uma testemunha. Assim, após tornarmos $N_{K}$ uma clique, basta verificar se o grafo resultante satisfaz tal condição necessária. Se o grafo resultante não satisfizer a condição necessária descrita pelo Teorema 1, uma nova partição deve ser considerada.

4. $N_{S}=\emptyset, N_{K} \neq \emptyset, P_{S} \neq \emptyset$ e $\left(N\left(P_{S}\right) \cap P_{K}\right)=\emptyset$. Como $P_{S}$ não tem vizinhança em $P_{K}$ e $G$ não é split bem-coberto, $P_{S}$ tem vizinhança em $N_{K}$. Neste caso, 
todos os vértices de $K$ têm que possuir testemunha e, como os vértices de $P_{K}$ não têm vizinhos em $P_{S}$ e $N_{S}=\emptyset$, é necessário (e suficiente) que exista um vértice $u$ em $N_{K}$ que servirá como testemunha para os vértices de $P_{K}$. Note que $u$ é adjacente a todos os vértices de $P_{K}$. Além disso, se, eventualmente, existirem outros vértices em $N_{K}$ que não estão na vizinhança de $P_{S}, u$ também servirá como testemunha para eles. Portanto, se a existência do vértice $u$ em $N_{K}$ for confirmada e adicionarmos arestas entre cada par de vértices de $N_{K} \backslash\{u\}$, basta verificarmos se o grafo resultante é split bem-coberto. Em caso negativo, existe um vértice em $K$ com mais de uma testemunha (não é possível ter vértice em $K$ sem testemunha, por construção). Observe que tais testemunhas estão em $P_{S}$, sendo inerentes, portanto, à partição considerada. Logo, neste caso, uma nova partição deve ser considerada.

5. $N_{S} \neq \emptyset, N_{K} \neq \emptyset$. Observe que, como $G$ não é split bem-coberto, em $N_{S} \cup P_{S}$ deve existir pelo menos uma testemunha para $N_{K} \cup P_{K}$ e, neste caso, todas as testemunhas pertencem a $N_{S} \cup P_{S}$. Sendo assim, após fazermos $G\left[P_{K} \cup N_{K}\right]$ ser uma clique, resta-nos verificar se o grafo resultante é split bem-coberto. Em caso negativo, como, por construção, o grafo é split, existem vértices em $K$ sem testemunha ou com mais de uma testemunha. Como as arestas adicionadas não acrescentam testemunhas, o problema de ter mais de uma testemunha está na partição considerada. Se, eventualmente, um vértice de $N_{K}$ ficou sem testemunha, este problema também se deve à partição, uma vez que todas as testemunhas estão restritas ao conjunto $N_{S} \cup P_{S}$. Logo, em ambos os casos, uma nova partição deve ser analisada.

Finalmente, checar se um grafo é split bem-coberto, toma $O(m)$ passos, o passo 8 que é o mais custoso, toma $O(n m)$, e o número de partições esparso-densas é $O\left(n^{2}\right)$. O que totaliza a complexidade de tempo $O\left(n^{3} m\right)$.

\section{Referências}

Alves, S. R., Dabrowski, K. K., Faria, L., Klein, S., Sau, I., and Souza, U. S. (2016). On the (parameterized) complexity of recognizing well-covered $(r, \ell)$-graphs. In COCOA 2016, Hong Kong, China, 2016, pages 423 - 437. LNCS.

Chvátal, V. and Slater, P. J. (1993). A note on well-covered graphs. In John Gimbel, J. W. K. and Quintas, L. V., editors, Quo Vadis, Graph Theory?, volume 55 of Annals of Discrete Mathematics, pages 179 - 181. Elsevier.

Feder, T., Hell, P., Klein, S., and Motwani, R. (1999). Complexity of graph partition problems. In Proceedings of the Thirty-first Annual ACM Symposium on Theory of Computing, STOC '99, pages 464 - 472. ACM, New York, NY, USA.

Golumbic, M., Kaplan, H., and Shamir, R. (1995). Graph sandwich problems. volume 19, pages $449-473$.

Golumbic, M. C. (1980). Algorithmic graph theory and perfect graphs. In Academic Press, volume 1, pages 149 - 156. Elsevier Science. 